\title{
Multi-Objective Optimization in High Frequency Electromagnetics-An Effective Technique for Smart Mobile Terminal Antenna (SMTA) Design
}

\author{
Junwei Lu, David Ireland, and Andrew Lewis \\ Griffith University, Nathan, Qld 4111, Australia
}

\begin{abstract}
This paper describes several major optimization techniques, such as FDTD-simulated annealing (SA), FEM-Genetic algorithms (GA), and FD-TD-particle swarm optimization (PSO) and frequency domain FEM-PSO used for high-frequency electromagnetics and their applications in smart mobile terminal antenna (SMTA) design. Two SMTAs, a dielectric embedded electronically switched multi-beam (DE-ESMB) antenna and a dielectric embedded electronically steerable passive array radiator (DE-ESPAR) antenna, were successfully developed by using these multi-objective optimization techniques. The design considerations and rules based on computational electromagnetics and optimization techniques are explored for the practical application of dielectric embedded SMTAs.
\end{abstract}

Index Terms-Dielectric embedded electronically switched multi-beam antenna, genetic algorithms, multi-objective optimization, particle swarm optimization, simulated annealing, smart mobile terminal antennas.

\section{INTRODUCTION}

$\mathbf{C}$ OMPUTATIONAL electromagnetic modeling techniques have moved from analysis to design in electrical and electronic engineering practice. Computational electromagnetics (CEM) with optimization techniques can be used to solve real-life, high-frequency, electromagnetic field, and wave propagation problems and help industry to provide better products, such as antennas and arrays. CEM with optimization techniques can truly help but, before it can be used effectively, the basic concepts of CEM modeling and optimization techniques must be understood.

Various novel smart mobile terminal antennas (SMTAs) have been developed for mobile wireless computing network and communications systems in recent years. Among them, the most significant achievements are the dielectric embedded electronically switched multi-beam (DE-ESMB) antenna [1], and dielectric embedded electronically steerable passive array radiator (DE-ESPAR) antenna [2], which exhibit many advantages such as low profile, small and compact size, and high performance. However, these SMTAs do not follow conventional antenna design rules due to the complexity of the antenna array structure associated with high dielectrics, and strong mutual coupling between antenna elements inside the dielectric material. Design of an SMTA is a multi-objective optimization problem which requires significant design considerations when CEM with optimization techniques is employed for full-wave solutions in either time domain or frequency domain.

This paper provides recommendations and guidance for the utilization of FD-TD and FEM-based full-wave techniques associated with several major optimization techniques, such as FDTD-simulated annealing, FEM-genetic algorithms, FD-TDparticle swarm optimization (PSO) and FEM-PSO for SMTA design. The differences between these approaches for designing dielectric embedded SMTA (DE-SMTA) are also discussed and several examples are given.

Manuscript received October 07, 2008. Current version published February 19, 2009. Corresponding author: J. Lu (e-mail: j.lu@griffith.edu.au).

Color versions of one or more of the figures in this paper are available online at http://ieeexplore.ieee.org.

Digital Object Identifier 10.1109/TMAG.2009.2012586

\section{DESIGN CONSIDERATIONS OF SMTAS}

\section{A. Full-Wave Computational Techniques}

In SMTA design, full-wave computational techniques are often used for antenna structure design and performance simulation. A full-wave computational technique provides a complete solution to Maxwell's equations within the computational space for all conductors and materials. Full-wave techniques are more complex than quasi-static techniques, but they are also generic in nature and have fewer limitations in their use. The limitations of practical full-wave techniques vary from technique to technique and depend on the level of detail of the model. Each full-wave modeling technique is limited to particular types of models.

In the application of full-wave techniques, the solution can be found in either the time domain or the frequency domain. Frequency-domain codes solve for one frequency at a time. This is usually adequate for antenna work and for examining specific issues. Frequency-domain codes are in general faster than their time-domain cousins. Therefore, several frequency-domain simulations can usually be run in the time it would take for a single time-domain simulation. A further benefit to using frequency-domain codes is their capacity to use larger meshes for the lower frequencies, which in turn permits a shorter computation time. However, to cover a wide frequency range with frequency-domain codes, a number of simulations are required.

Unlike conventional smart antenna arrays, in DE-SMTA, the separation of distances between elements can be reduced to $0.25 \lambda_{0}$ [3]. To further reduce the SMTAs' physical size for the portable application, the antenna arrays must be embedded in a high-dielectric material. However, the conventional antenna design concept made DE-SMTA design impossible due to the complexity of the antenna array structure associated with embedded dielectric material and air interface. Moreover, the design technique and theory for these SMTAs are not available. Therefore, the only way to achieve an optimal antenna design is to use a full-wave numerical solution and control it with a robust optimization algorithm.

\section{B. DE-SMTA Arrays Using Time-Domain Approach}

One of the most popular CEM techniques, the FD-TD method, has been used, as it is well suited to modeling complex objects, being able to include finite dielectric media, thin wires, 


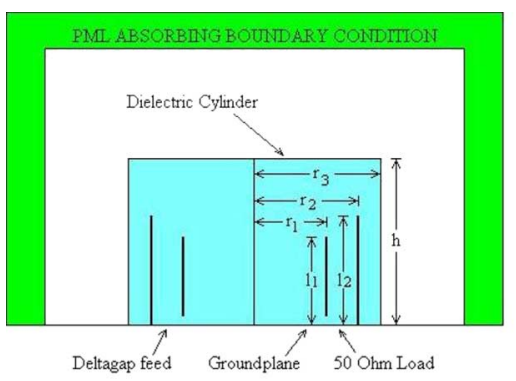

(a)

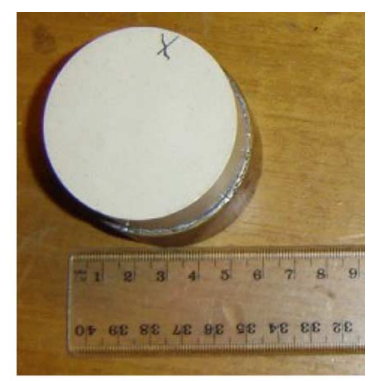

(b)
Fig. 1. Simulation domain of DE-ESMB including white space and PLM: (a) cross section of cylindrical structure of DE-ESMAB and (b) working prototype of DE-ESMAB with 1/4 wave length grounding skirt.

and lumped loads. Parameters of interest that can be derived from FD-TD simulations include broadband input impedance, radiation patterns, and time harmonic field strengths. A system of six coupled partial differential equations in the Cartesian coordinate system forms the basis of the FD-TD numerical algorithm for electromagnetic wave interactions with general three-dimensional objects [4]. The time step was set to the Courant stability limit.

\section{DE-SMTA Arrays Using Frequency Domain Approach}

The FEM in the frequency-domain technique has been employed for the simulation domain, where the frequency-domain vector wave equation for the $\boldsymbol{E}$ field can be derived as

$$
\nabla \times \frac{1}{\mu} \nabla \times E+\sigma_{e} \omega E+\omega^{2} \varepsilon E=-j \omega J
$$

where $\omega$ is angular frequency, $\boldsymbol{J}$ is source current, $\sigma_{\mathrm{e}}$ is the effective conductivity, and $\mu$ and $\varepsilon$ are the permeability and permittivity of the problem space, respectively. Similar parameters of interest as those mentioned in Section II-B can be derived.

\section{Simulation Domain and Boundary Conditions}

Since in antenna modeling problems, the incident radiation cannot be described as a plane wave, an effective boundary condition must be considered for the open boundaries. The perfectly matched layers (PMLs) provide good performance for a wide range of incident angles and are not very sensitive to the shape of wave fronts [5]. Fig. 1 shows the cross section of the DE-SMTA computation model with cylindrical PML boundaries for the FD-TD based simulation model. Spherical PML boundaries are also used for the FEM frequency-domain simulation, where the minimum distance between the DE-SMTA and boundaries is around 1/2 wavelength at the operating frequency.

\section{Multi-ObJective Optimization AlgORIthMS FOR DE-SMTA DESIGN}

\section{A. Multi-Objective Optimization Problems in DE-SMTAs}

Various optimization techniques have been introduced to computational electromagnetics, among them the most successful and widespread optimization techniques are the GA and PSO [6], [7]. The GA differs from PSO; the GA is based on genetic encoding and natural selection, with a mutual exclusivity that leaves open the possibility of integrating the two techniques, while PSO is based upon social swarm behavior.

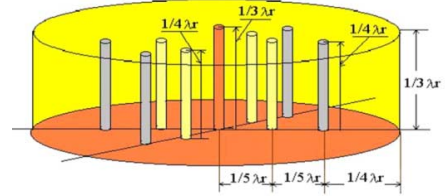

(a)

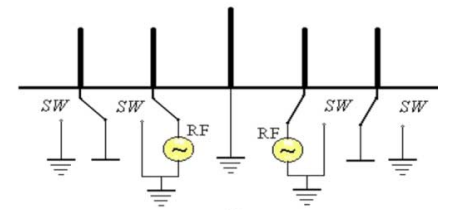

(b)
Fig. 2. DE-ESMB simulation using FD-TD-based time-domain approach: (a) configuration of DE-ESMB and (b) the connection of antenna elements to the switching circuit.

Obviously, these two optimization techniques rest upon different paradigms. One advantage of PSO over the GA is its algorithmic simplicity [8]. The GA consists of three major operators: selection, crossover, and mutation. In PSO, there is only one simple operator: velocity calculation.

To fully explore the DE-SMTA performance in wireless communications networks, optimal design is often required, as the size of the antenna array and the material of the dielectric directly affect the antenna performance. For a portable antenna used in wireless communications and computing networks, small size is critical. The size reduction factors can be expressed as a function of the shape, size, and electrical characteristics of the dielectric material and the location of each wire element in the material. Thus, the actual reduction factor of DE-ESMB, $F_{r a}$, for the size of antenna will be

$$
F_{r a}=f\left(\sqrt{\varepsilon_{r}}, h, r_{1}, r_{2}, r_{3}, l_{1}, l_{2}\right)
$$

where $h$ is the height of dielectric, $r_{1}$ is the radius of inner element circle, $r_{2}$ is the radius of outer element circle, $r_{3}$ is the radius of embedding dielectric, $l_{1}$ the length of the elements on the inner circle, and $l_{2}$ the length of the elements on the outer circle, respectively. This size reduction factor must be considered in the optimization simulation.

Optimal design in electromagnetic product development is often concerned with defining or determining the objective functions and constraints. Since the DE-SMTA design is a typical multi-objective optimization problem, a number of aspects are required in the design, such as antenna gain and desired radiation pattern, reflection coefficient and size. However, without prestudy of the simulation model, one cannot find the solution model quickly, due to the complex structure and many other design constraints. The required antenna resonant frequency and electrical characteristics may not appear at the minimum antenna size or volume. Therefore, this kind of simulation problem can be considered also as a constrained optimization problem. The objective functions and constraints should be defined before introducing the optimizing design process to DE-SMTA design.

\section{B. FD-TD-SA for DE-ESMB Antenna}

The DE-ESMB antenna consists of multi-feed elements surrounded by a ring of parasitic elements as directors or reflectors and was embedded in a high-dielectric-constant material as shown in Fig. 2. The size of the simulation domain was $120 \times$ $120 \times 60$ cells with a cubic space discretization of $1 \mathrm{~mm}$ per cell.

In an early application of optimization, several DE-ESMB antenna arrays were successfully developed using FD-TD with simulated annealing, where size reduction, antenna gain, desired radiation pattern, and directivity were obtained. Based on the initial approach of defining the objective functions and constraints, a number of DE-ESMB antenna arrays were optimized, using the FD-TD computation method associated 

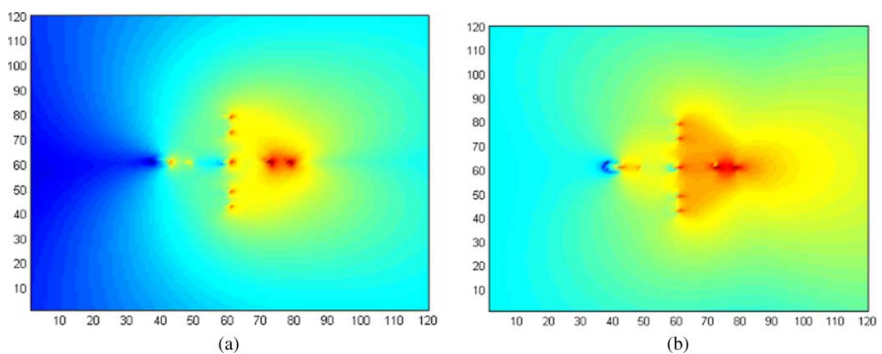

Fig. 3. Distribution of normalized $\mathrm{E}$ and $\mathrm{H}$ fields in $x, y$ plane in the crosssectional DE-ESMB antenna array above the ground plane. (a) $\mathrm{H}$ field; (b) $\mathrm{E}$ field.

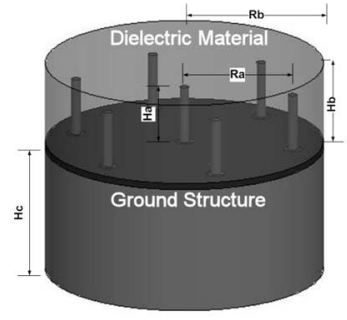

(a)

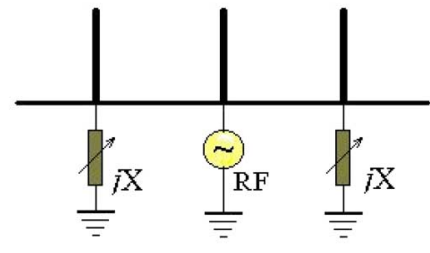

(b)
Fig. 4. DE-ESPAR simulation using FEM based frequency domain approach: (a) Configuration of DE-ESPAR and (b) beam control circuit.

with the adaptive simulated annealing (ASA) optimization algorithm [9], with physically constrained input dimensions such as the antenna height $\boldsymbol{h}$ and the element length $l_{i}$, the distance of antenna element to the central element $r_{i}$, and so forth. The objective function for the DE-ESMB antenna array was defined as an average reflection coefficient from 2.4 to $2.5 \mathrm{GHz}$ of $-25 \mathrm{~dB}$ and a front-to-back (FB) ratio of $20 \mathrm{~dB}$.

$$
\mathrm{Obj}=\left(25+S_{11} \cos t\right)+\left(20-R_{f b} \cos t\right) .
$$

After 92 iterations of the simulation, an objective function result of 13.94 was obtained with the physical size of the model shown in Fig. 2. The results of near field are presented in Fig. 3 where $\boldsymbol{H}$ field and $\boldsymbol{E}$ field indicate the distribution of the EM field inside the dielectric material.

\section{FEM-GA for DE-ESMB and DE-ESPAR}

The same optimal design technique can be applied to the DE-ESMB and DE-ESPAR designs shown in Figs. 2 and 4. The 13-element DE-ESMB shown in Fig. 2 was designed and optimized using a FEM-based full-wave technique in the frequency domain, associated with a GA. To achieve the best beam-forming performance, the high-frequency structure of the DE-SMTA antenna, various antenna properties and the reactance of the beam control circuit were optimized. However, frequency-domain FEM with GA-based simulation was very slow for both DE-ESMB and DE-ESPAR when a frequency swapping technique used. It almost took one week with a normal workstation to achieve the desired result. Therefore, the faster computation techniques or parallel computations with optimization tourniquets [A1] are suggested for practical DE-SMTA design.

The optimized results for a DE-ESMB antenna with 13 elements are presented in Fig. 5, where the expected results of reflection coefficient $\mathrm{S}_{11}$, and better antenna performance have been achieved by using such GA-based optimal design.

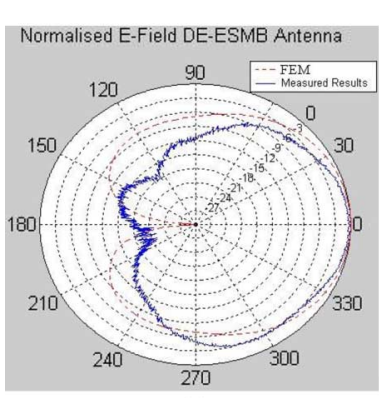

(a)

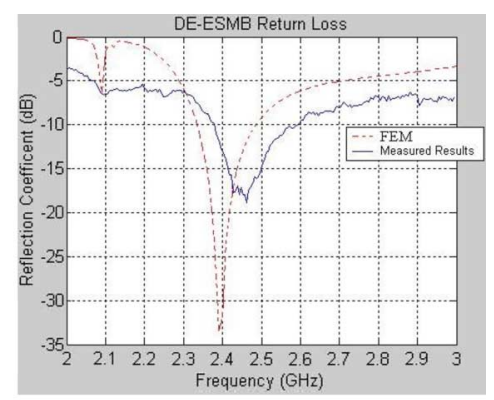

(b)
Fig. 5. Comparisons of FEM-GA based computation results and experiment results for a prototype DE-ESMB antenna with 13 elements: (a) radiation pattern and (b) reflection coefficient.

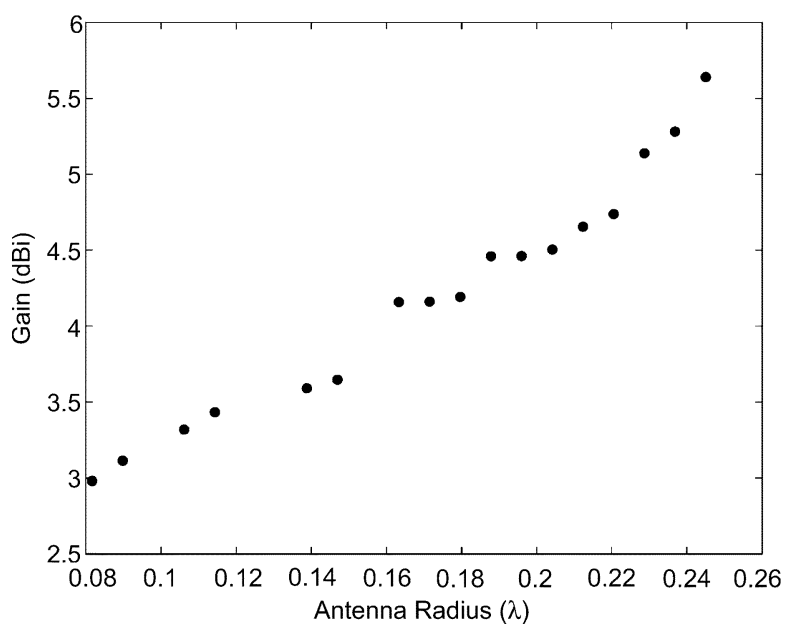

Fig. 6. Pareto front for antenna gain versus antenna radius based on the FD-TDPSO.

\section{FD-TD-PSO for DE-ESPAR}

Particle swarm optimization (PSO) has been found to be effective in optimizing difficult and multi-dimensional problems in a variety fields. Recently, this technique has been successfully applied to antenna design. The advantage of PSO over GA is its algorithmic simplicity leading to a reduction of computation and elimination of the necessity to select the best operating parameters for a given problem. PSO also has the ability to control convergence of the computation process. The most profound advantage of the PSO algorithm is its inherent simplicity while offering exceptional performance relative to other conventional methods.

Modeling of the antenna structure was achieved using the finite-difference time-domain method. In order to model the ON/OFF states of the parasitic elements, an insulation region was defined in the model which separated the element from the ground plane simulating an OFF position. For the ON position, the parasitic element was directly connected to the ground plane. Two objective functions were used in the optimization process, with the first being

$$
f_{1}=\left(1-\left|S_{11}\right|\right) D
$$

where $D$ denoted the directivity of the antenna. Assuming no other losses, this equation corresponds to the absolute gain of the antenna. The second objective function $f_{2}=f(r)$ was simply the radius of the dielectric material.

Searching and converging to the Pareto-front can be done using a plethora of optimization algorithms. In this instance, 


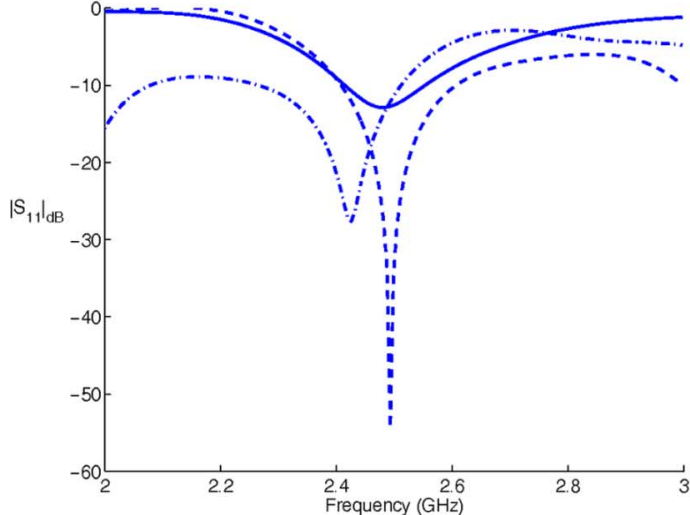

(a)

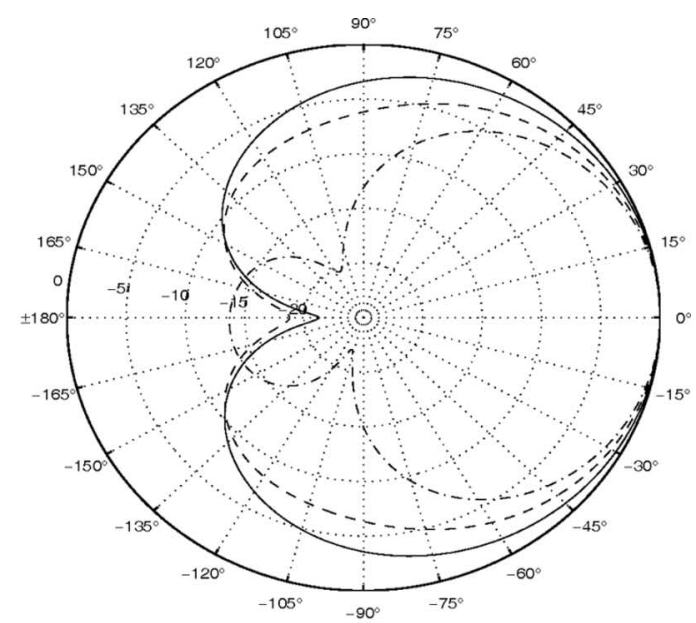

(b)

Fig. 7. Computation results of the FD-TD-PSO: (a) reflection coefficient and (b) radiation patterns for different antenna size.

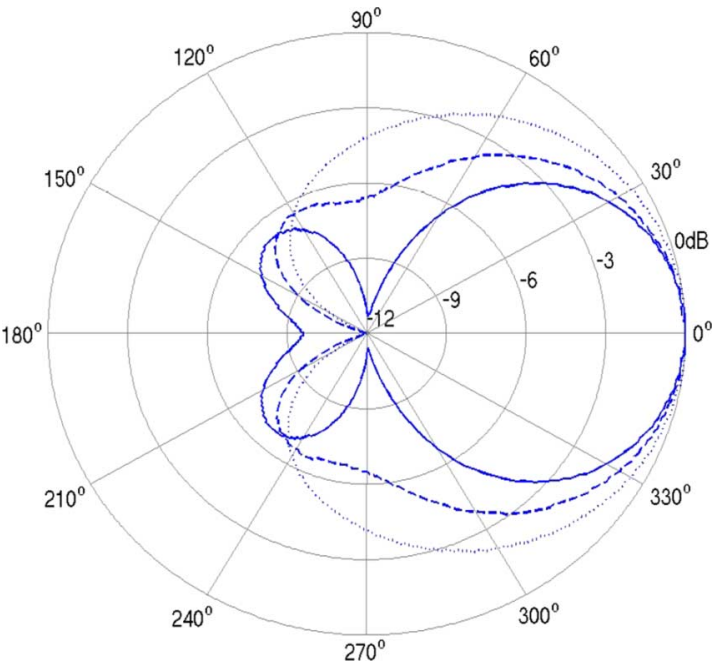

Fig. 8. Computation results of the FEM-PSO, radiation patterns for different antenna size, where _ _ is the antenna which had the best gain but largest size, --- is the antenna which had a compromise between gain and size, ..... is the antenna which had a low gain but small size.

however, the PSO algorithm was deployed, and the computation results of the set of (Pareto) optimal solutions for antenna gain and antenna radius, and the reflection coefficient and radiation patterns for three different antenna sizes are shown in Figs. 6 and 7, respectively.

The dimensions of the antenna structure that were varied included the height of the central active element $h_{a}$, the height of the parasitic elements $h_{b}$, the radius of the parasitic array $r_{a}$, and the toggle states of the parasitic elements. The radial size of the antenna structure was kept $3 \mathrm{~mm}$ greater than the array radius. The ground skirt was kept constant at $0.25 \lambda$.

\section{E. FEM-PSO for DE-ESPAR}

Frequency-domain FEM-PSO has been found to be a faster and more efficient modeling technique in DE-ESPAR design as several frequency-domain simulations can usually be run in the same time as a single FD-TD simulation. The computation time is much shorter than FD-TD-PSO under the same initial approach of defining the objective functions as (4) and constraints.
Moreover, the frequency-domain FEM-PSO has the capacity to use larger meshes for the lower frequencies, which in turn permits a shorter computation time. The calculated radiation patterns for optimized antenna size are shown in Fig. 8.

\section{CONCLUSION}

While multi-objective optimization in DE-SMTA design greatly improves the resulting antenna designs, the methods used must be carefully chosen. From the experiences of the authors, it is suggested that when using a time-domain approach, the FD-TD with SA or GA can be used, while FEM with PSO is recommended for frequency-domain applications as preconstraints will prevent a wide frequency sweep and therefore the computation time can be significantly reduced. The same recommendation is applicable to design of other antenna arrays and small antennas, as it is more a function of the solution method than the antenna structure. It has been noticed also that there is very little variation in bandwidth for this particular antenna structure.

\section{REFERENCES}

[1] J. Lu, D. Thiel, and S. Saario, "FD-TD analysis of dielectric embedded electronically switched multiple-beam antenna array," IEEE Trans. Magn., vol. 38, no. 2, pp. 701-704, Mar. 2002.

[2] J. W. Lu, D. Ireland, and R. Schlub, "Dielectric Embedded ESPAR (DE-ESPAR) antenna array for wireless communications," IEEE Trans. Antennas Propag., vol. 53, no. 8, pp. 2437-2443, Aug. 2005.

[3] R. Schlub, J. Lu, and T. Ohira, "Seven element ground skirt monopole ESPAR antenna design from a genetic algorithm and the finite element method," IEEE Trans. Antennas Propag., vol. 51, no. 11, pp. 3033-3039, Nov. 2003.

[4] A. Taflove, Computational Electromagnetics, The Finite-Difference Time-Domain Method. Norwood, MA: Artech House, 1995.

[5] J. Jin, The Finite Element Method in Electromagnetics, 2nd ed. New York: Wiely-IEEE Press, May 2002.

[6] D. S. Weile and E. Michielssen, "Genetic algorithm optimization applied to electromagnetics: A review," IEEE Trans. Antennas Propag., vol. 45, pp. 343-353, Mar. 1997.

[7] J. Kennedy and R. Eberhart, "Particle swarm optimization," in Proc. IEEE Conf. Neural Networks, vol. IV, pp. 1942-1948.

[8] J. Robinson and Y. Rahmat-Samii, "Particle swarm optimization in electromagnetics," IEEE Trans. Antennas Propag., vol. 52, no. 2, pp. 397-407, Feb. 2004.

[9] Adaptive Simulated Annealing Method. [Online]. Available: www.ingber.com 